\title{
Multidisciplinary approach in child and adolescent depression: Experience from a tertiary mental health institution in India
}

\author{
Deepak Kumar $^{1 *}$, Uday K. Sinha ${ }^{2}$, Amit Khanna ${ }^{1}$, Sujit Kumar Kar ${ }^{1}$ \\ ${ }^{1}$ Department of Psychiatry, Institute of Human Behaviour and Allied Sciences, Delhi, India \\ ${ }^{2}$ Department of Clinical Psychology, Institute of Human Behaviour and Allied Sciences, Delhi, India \\ Email: srivastav.deep@gmail.com
}

Received 27 March 2013; revised 30 April 2013; accepted 7 May 2013

Copyright (C) 2013 Deepak Kumar et al. This is an open access article distributed under the Creative Commons Attribution License, which permits unrestricted use, distribution, and reproduction in any medium, provided the original work is properly cited.

\begin{abstract}
The concept of Multidisciplinary approach is not new to Medical Science. It is an evolving concept that is widely appreciated and adopted in most of the disciplines of medicine. It's rationale and applicable in the management of Child and Adolescent Depression, which needs to be revisited focusing on the interplay of various contextual factors that are entwined and tend to affect the course of the illness. The limitations of any one mental health professional in envisioning all possible dimensions of Child and Adolescent Depression make this approach all the more important wherein Psychiatrists, Clinical Psychologists, Psychiatric Social Workers collaborate with each other and other allied professionals in the treatment and management of the disorder. India, being a heavily populated country, where multidisciplinary management approach is in a phase of expansion, is practiced in many leading institutes to meet the quality needs of patients. This article focuses on the Indian scene of multidisciplinary management approach in child and adolescent depression.
\end{abstract}

Keywords: Child and Adolescent Depression; Multidisciplinary Approach; Indian Scene

\section{INTRODUCTION}

The McGraw-Hill Concise dictionary of Modern Medicine defines "Multidisciplinary approach as the philosophy of converging multiple specialties and/or technologies to establish a diagnosis or affect a therapy” [1]. Various contextual factors tend to affect medical as well as psychiatric disorders and so the practice of Medicine

"Corresponding author. has over time, gradually they evolved and inculcated the paradigm of multidisciplinary approach.

The concept of multidisciplinary approach may appear very difficultly and complicatedly, but in reality it is nothing more than having observations and interpretations from different viewpoints by professionals coming from different fields of science and their coordination to generate a holistic picture of the problem in question and its treatment solutions. This is essentially based on the Biopsycho-social model in which the biological, psychological and the social factors causing an illness are dissected, analyzed and modulated as per the existing scientific understanding to bring about a change in the course of the illness [2].

Multidisciplinary approach is a noble approach modality, which brings multiple related disciplines into action on one platform for solving a complex issue in a simplified manner. It is a holistic approach, which covers the global aspects of the problem rather than a focal issue. This approach not only holds valid for health care in general but also to many other important domains like education, occupation, management as well as administration. The management approaches of individual professionals who may not be competent and skilled enough to focus on every aspect of illness lasted for centuries together without much resistance. But development of science over generations has availed highly skilled professionals with the capacity to analyze different finer aspects of complex issues and bring about a global change.

This approach is all the more important when it comes to management of individuals with disability as a result of illness, affecting multiple domains of a life. In such cases, simply treating the illness with drugs is not enough in overcoming the deficits and expertise of various professionals in the field of rehabilitation is required 
[3-7]. When there is a need to meet multiple goals in the domains of health and functioning while treating an illness, a collaborative approach of multiple disciplines is an appropriate choice. The goal achieved by the multidisciplinary approach is unlikely to be achieved by the approach of individual health professionals [5,6].

Multidisciplinary management approach has the following advantages $[3,4,8]$.

1) Patient's positive perception about the quality of care;

2) Availing care as per practice guidelines;

3) Availing standard care;

4) Client satisfaction;

5) More informative care;

6) Better psycho-social support and practice support.

The limitations with multidisciplinary management approach is that it is not available everywhere, not accessible to everyone and of course not affordable by everyone. In all underdeveloped and most developing countries, where elementary health care services are the basic needs, multidisciplinary management approach is beyond reach.

\section{MULTIDISCIPLINARY APPROACH IN MEDICAL DISORDERS}

A famous quote "The eyes see what the mind knows" perhaps best can explain the importance of making inferences by professionals from different disciplines as one alone may not be able to perceive all plausible causes of the problem. Multidisciplinary approach is not new in medical disorders; rather the concept was first introduced and more frequently required for management of medical disorders.

Obesity is quite common now days, and is frequently associated with co-morbidities like diabetes, hypertension, cardiovascular diseases, arthritis and leads to poor quality of life. Multidisciplinary approach carries a lot of significance in this context as it needs joint approach of surgeons, physicians of multiple specialties, dietician, physiotherapist etc. [9]. Similarly management of cancer is a challenging issue which needs a team of Primary Physician, Radiologist, Pathologist, Surgical Oncologist, Anesthesiologist, Medical Oncologist, Radiation Oncologist, Gynecologic Oncologist, Pediatric Oncologist, Psycho-oncologist, Rehabilitation Specialist, Nurse Oncologist, Oncology Pharmacist and other Support Personnel [10]. The same principle is also used in treating malignancies like rectal cancer, gastric cancer and preferred over other management approaches [11,12]. The therapeutic success depends largely on interdisciplinary coordination [10]. Laboratory professionals spread their roots to almost all specialties and frequently become a part of multidisciplinary team [13]. Multidisciplinary care, helps in developing treatment protocols, improves service co-ordination and detects better the emotional as well as psychosocial needs of cancer patients and ensures continuity of care [14]. Hand hygiene is basic need to prevent cross infections in hospitals but rarely the practice guidelines are followed routinely and multidisciplinary approach is helpful in improving adherence to hand hygiene practice guidelines [15]. Multidisciplinary approach by team of clinician, radiologist and pathologist in evaluating cases of idiopathic interstitial pneumonia was found to be a more accurate diagnostic approach for the disease entity [16].

\section{IMPORTANCE OF MULTIDISCIPLINARY APPROACH IN PSYCHIATRIC DISORDERS}

Multidisciplinary approach is the need of the hour in effectively treating patients with severe mental illnesses, particularly in community settings [17]. Comprehensive care for such patients is possible with the collective efforts of Psychiatrists, Clinical Psychologists, Psychiatric Social Workers, Psychiatry Nurses, Occupational Therapists and Physiotherapists. Liaisoning of mental health team in medical emergency department has been instrumental in effective crisis management in patients suffering from psychiatric disorders especially those who are victims of abuse and trauma [18]. Patients suffering from psychiatric disorders are at more risk for physical illnesses like cardiovascular disorders, diabetes, because of the adverse effects of psychotropic medications. They are also at higher risk for suicide, deliberate self-harm and accidents. In view of this strong association between psychiatric illnesses and physical illness liaisoning between physician and psychiatrist can improve the quality of life in these patients [19]. Similarly chronic physical illnesses have psychiatric sequel which again requires collaborative approach between physician and psychiatrist.

Substance abusers not only need pharmacotherapy regimen prescribed by the psychiatrists, but also need support of clinical psychologist for motivation enhancement, brief intervention and guidance of psychiatric social worker for integration with community and occupational rehabilitation. Role of psychiatric nurse and for management of physical complications, intervention by physician are equally important. A multidisciplinary approach, as a holistic approach gives the best results in de-addiction practice.

\section{MULTIDISCIPLINARY APPROACH TO CHILD AND ADOLESCENT DEPRESSION}

The complex and multi-factorial nature of childhood depression involving various genetic, individual and environmental factors poses a unique challenge often re- 
quiring the expertise of various clinical professionals in its management. Depression in child and adolescentpopulation can express itself through infancy to late adolescence with myriad presenting symptoms. Subtle signs such as rejection of feed by an infant and academic decline in a school going child requires an astute eye to recognize it as a symptom of depression and negate other conditions.

Childhood depression affects $2.5 \%$ of children up to 12 years of age and up to $8.5 \%$ in adolescence causing significant socio-occupational dysfunction [20]. With the growing urbanization and stressors of daily living the number is likely to rise, thereby making it all the more important for childhood depression to be identified at the earliest and treated. Multidisciplinary approach is required not only at the time of assessment but throughout the course of its treatment [21]. The family members form the most important support system for the child and can provide important information related to behavioral change which brings in the role of the multidisciplinary team at the outset. The child may be brought by the family to a general physician or a pediatrician or to a mental health professional. In case of schools the role of teachers in recognizing withdrawn behavior, decreased socialization and poor academic performance as early symptoms of an underlying psychological problem cannot be under stated. In residential setups, the care taker may bring the child to a mental health professional. The initial assessment may be done by a Psychiatrist or allied mental health professionals such as Clinical Psychologists and Psychiatric social workers. On many occasions, exact measurement of the depressed mood state is needed which is done using various rating scales and inventories which require the assistance of a Clinical Psychologist. The Figure 1 explains the ideal multidisciplinary approach in child and adolescent depression. After identification and assessment of depression in a child, he/she may be referred to a psychiatrist or clinical psychologist or any specific mental health care provider for specific issues, but the ideal approach is being referred to the multidisciplinary team, subject to its availability.

The initial stages of assessment of childhood depression are most important for sharing information between clinicians and understanding the risk and the protective factors which would assist in the holistic management of the child. The exchange of information related to various medical and psychosocial areas of the child between different clinicians can happen over forums such as service rounds and interdisciplinary rounds, in outpatient department thereby facilitating a multidisciplinary approach in management. Mild to moderate depression usually re-

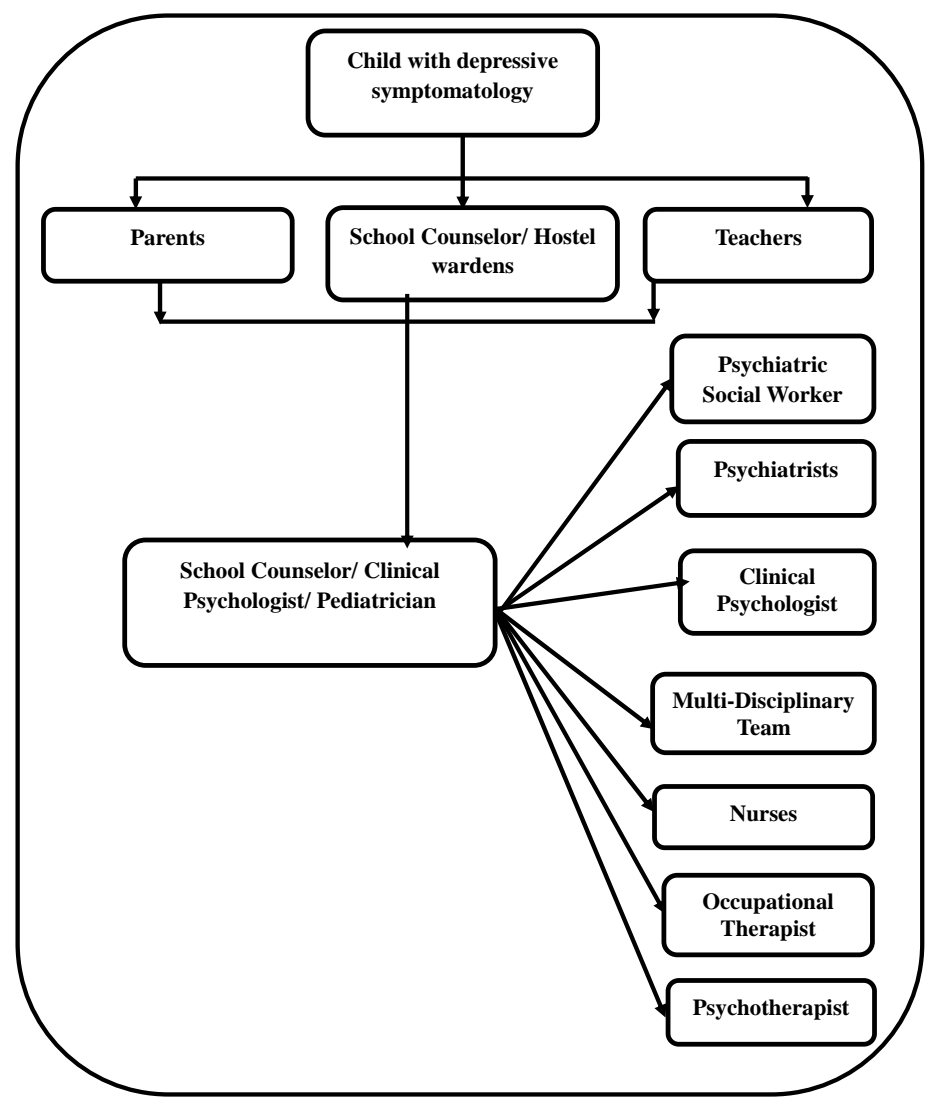

Figure 1. Multidisciplinary management in child and adolescent depression. 
quire supportive psychotherapy or Cognitive Behavioral Therapy which is provided by Clinical Psychologists and Psychiatric social workers and severe depression with or without suicide risk require pharmacotherapy by a Psychiatrist based on the best available scientific evidence. Although Modified Electroconvulsive Therapy is required in few cases, but its use is controversial in child and adolescent age group especially under the new Mental Health Care Bill in India.

Assessment and management of depression in children living in residential care setups requires inter-sectoral collaboration with various legal and quasi legal agencies along with medical and mental health professionals. Once the acute stage of treatment is over, the child requires continued support and guidance through the multidisciplinary mental health team. At this stage and further in life, the services of the educationist, occupational therapist and physiotherapist may be required in case of children suffering from co-morbid physical disability.

Multidisciplinary approach should come as a spinal response from those planning and organizing mental health services for children. If each professional is prompt, a comprehensive plan of management can be formulated which would go a long way in treatment of childhood depression from assessment to restoration.

\section{CASE STUDIES}

\subsection{Case 1}

A 16 years old adolescent boy had presented to the child guidance clinic with sadness of mood, withdrawn behavior, poor scholastic performance, feelings of guilt, disturbed sleep, and poor appetite for six months. He was also having multiple somatic complaints like-headache, constipation, bodyache, lethargy, fatigability. On further exploration, the patient had revealed his guilt feelings related to past acts of masturbation and attributing his depressive and somatic symptoms to loss of semen. Patient's family members, particularly his grandmother had extreme negative view about masturbation. He received a lot of critical comments and harsh punishment for doing masturbation in the past. The boy was reported as shy and introvert in his temperament. He was diagnosed to be suffering from severe depressive episode. Appropriate antidepressant therapy was started. Family's punitive attitude towards patient's masturbation and sexual myths were addressed by the team of psychiatrists, clinical psychologists and psychiatric social workers. The patient showed significant response within a short period to this multidisciplinary approach.

\subsection{Case 2}

A 14 years old boy, a primary school dropout belonging to a middle socio-economic status family was admitted in the child and adolescent psychiatry unit. He was having complaints of sadness of mood, poor social interaction, crying spells, death wishes and suicidal attempts. He was also having reduced sleep, poor appetite, and poor self-care. He had lost his interest in watching television and playing with friends. These symptoms were constantly persisting for approximately three months prior to hospitalization. On detailed probing, his mother reported that he used to engage himself in cross dressing when he was alone at home since eight years of age. He used to wear his sister's dresses and used to do it despite family members efforts to prevent him from doing so. There were no masturbatory or erotic self-stimulatory activities to obtain gratification. He often said that he wanted to be a girl and felt disgusted with himself for being a boy. He believed himself to be a female and dreamt of marrying a male and experience pregnancy. He had multiple homosexual contacts two years prior to hospitalization. He was diagnosed with a severe depressive episode with suicide risk with gender identity disorder. He was approached by the team of psychiatrists, clinical psychologists and psychiatric social workers. Antidepressant (Fluoxetine) was started. High risk management, strict vigilance in view of risk of suicide was ensured. Family was addressed about the other high risk behaviors like unprotected homo-sexual activities. Behavior therapy sessions were conducted. The patient had shown satisfactory improvement to the multidisciplinary approach of management and is on regular follow up.

\section{RELEVANCE OF MULTIDISCIPLINARY APPROACH IN INDIA}

There is scarcity of research works regarding multidisciplinary management approach in child \& adolescent depression in India. Child and Adolescent Psychiatry (CAP) services in India are provided by Private and Public sector alike. In India, specialized services in Child and Adolescent psychiatry are provided by Child Psychiatrist/Psychologists. These professionals have either spent years working in this field or have a fellowship or D.M degree in Child and Adolescent psychiatry. The latter are in small numbers given the fact that specialized training in this field having commenced only recently and not available across the country.

Given the high burden of mental illness in child and adolescent population, the ground reality is that most cases with psychological/psychiatric problems first present to a pediatrician. The Pediatrician has a unique opportunity in being easily accessible to the family, is more aware of the ecosystem of the family and the child and his services are provided without the family or child perceiving any stigma of having a mental illness. With basic training in mental health issues and its management 
in the pediatric age group, the pediatrician can serve as an important link in the multidisciplinary management of psychiatric/psychological illnesses in children with the assistance of a Clinical Psychologist and refer difficult cases to a Child Psychiatrist. To improve multidisciplinary management in child and adolescent population, the role of a psychiatrist would lie in training of general physicians and pediatricians which will improve not only the quality of care and treatment given to the child but also increase the referrals to child and adolescent mental health units. Similarly, few of the elite schools (in urban areas) have availability of school counselors/psychologists who also form key referrals for emotional and behavioral disorders. Collaboration of non-government organizations to the specialized centers for detailed evaluation and therapies also facilitates multidisciplinary approach.

The availability of allied mental health professionals is not uniform across the country and across various Child and Adolescent Psychiatry units thereby hampering multidisciplinary management. According to the Urban Mental Health Project [22], the deficit of Clinical Psychologists and Psychiatric social workers is as high as $70 \%$. Data is not available on the deficit of these allied professionals in Child and Adolescent Psychiatry. This poses a significant problem in devising and strategizing multidisciplinary services in mental health care of children but at the same time should not be an impediment to the continued efforts to bridge gaps across disciplines where ever possible.

\section{CHILD AND ADOLESCENT MENTAL HEALTH SERVICES AT IHBAS, DELHI (INDIA)}

Institute of Human Behavior and Allied Sciences (IHBAS) is a Tertiary Neuro-Psychiatric Care Institute situated in the capital city of Delhi in India. It is the first Tertiary care Neuro-Psychiatric hospital in India in public sector with National Accreditation Board for Hospitals \& Healthcare Providers (NABH) accreditation. IHBAS has been providing specialized services in psychiatry, neurology and neurosurgery and dealing with potential medicolegal and court cases within the jurisdiction of Delhi and its neighboring states.

The hospital has an indoor capacity of 336 beds (including ten beds for Child and Adolescent Psychiatry) and is in the process of further expansion. Specialized OPD services are provided by the hospital and these include Child and Adolescent Psychiatric Clinic, Mental Retardation Clinic, Drug De-Addiction Treatment and Rehabilitation Clinic, Movement Disorder Clinic, Tobacco Cessation Clinic, Marital \& Psychosexual Clinic, Epilepsy Clinic, and Neurobehavioral Clinic.

The collaboration of IHBAS with the Child Welfare Committee's (CWC) and Juvenile Justices Boards since the beginning of Child ward in June 2009 has been very encouraging. The CWC's with its armamentarium of various non-governmental organizations and child helpline personnel have been very instrumental in bringing children in need of care, protection and treatment to IHBAS for various forms of psychological and psychiatric treatments. The treatment provided is by multidisciplinary team consisting of Psychiatrist, Clinical Psychologist and Psychiatric social worker, psychiatric nurses, occupational therapists and others.

There is a specialized Child and Adolescent Psychiatry ward which is ten bedded, shortly to be upgraded to 20 beds. Service providers form a team consisting of the following departments:

1) Psychiatry: A faculty, a senior resident and junior resident(s);

2) Clinical Psychology: Faculty/Non-faculty clinical psychologist, M Phil (Clinical Psychology) trainees;

3) Psychiatric Social Work: Faculty/Non-faculty psychiatric social workers;

4) Nursing staff: Sister in charge, staff nurses.

Additionally, services are availed from the departments of neurology, dietetics, occupational therapy, physiotherapy. For general medical/surgical conditions the children are referred to nearby general hospitals.

Detailed work-up of all new admissions is done within 24 hours by M Phil Clinical Psychology trainees or Psychiatry residents and all patients are seen by consultant in service rounds thrice a week and there is a multidisciplinary grand round once in a week.

There is a provision for family members to stay along with the children during admission. The ward provides an activity room having play items and for recreational activities. Children can also use them for reading and drawing. Structured program is provided throughout the day supervised by the Nursing staff, Clinical psychologists and Occupational therapist. There is also provision for assessment and therapies by Clinical Psychologists and Psychiatric Social Workers. All children are provided a multidisciplinary intervention to a degree required in each case. Daily meetings are also conducted with the parents providing them with general education and specific tips on parenting, behavioral modification etc.

We commonly encounter cases of childhood and adolescent depression in child guidance clinic. The outpatient services of child psychiatry specialty at IHBAS follow a multistep approach for Child and Adolescent Psychiatric disorders. Immediately following registration in the child guidance clinic, patients undergo a rapid screening process in the walk-in clinic carried out by a psychiatrist. After screening and detailed assessment, appropriate pharmacotherapy is prescribed to the patients. The Clinical Psychologist and Psychiatric Social Workers draw important inferences related to the temperament and psychosocial factors and share the inputs with the 
team.

Gautam Buddha once said "No deed is a deed till it's done for a higher cause”. Multidisciplinary management is not only the independence of various mental health professionals within the group but more importantly the cumulative and collective action taken after joint decision making to bring about the best outcome for the patient. It requires professionals to collaborate with each other unhesitatingly in the best interest of the patient and despite the ever increasing workload is possible to achieve.

\section{CONCLUSION}

Multidisciplinary approach is a need of the era. Collaborative relationship in multidisciplinary approach encourages team work and highly specialized skills of different specialties work together to deliver the best quality service. The number of members in the team depends on the availability and co-ordination between allied specialties. The team is usually led by a leader, who usually maintains co-ordination and facilitates the delivery of services. Multidisciplinary management, particularly in the context of adolescent depression is carried out by the team of psychiatrist, psychologist, psychiatric nurse and social worker. The role of a Psychiatrist is a pivotal role. Psychiatrist not only diagnoses and prescribes pharmacotherapy but also identifies different risk factors, psychosocial aspects and other complex issues and co-ordinate with other mental health professionals. The success and failure of multidisciplinary approach depends on the availability, assembly, association and the affirmation of resources at every step. Lapse at any of these levels can negatively affect the quality of the service and hence the role of a Psychiatrist becomes all the more important. The future role of a Psychiatrist is not only in training and research but also in leading professionals of various mental health disciplines and it is of paramount importance that this quality is inculcated in our practice and that we impart the same to the next generation of trainees.

\section{REFERENCES}

[1] Segen, J.C. (2002) McGraw-Hill concise dictionary of modern medicine. The McGraw-Hill Companies, Inc., New York.

[2] Engel, G.L. (1997) The need for a new medical model: A challenge for biomedicine. Science, 196, 129-136. doi:10.1126/science. 847460

[3] Agree, I. (2013) Palliative care curriculum for undergraduates (PCC4U): Funded by the Australian government, department of health and ageing.

http://www.pcc4u.org/index.php/learning-modules/focustopics/multidisciplinary-care/1-principlesof-a-multi-disciplinary-approach/activity-1-what-is-a-mul ti-disciplinary-approach-to-care
[4] Kuziemsky, C.E., Borycki, E.M., Purkis, M.E., Black, F., Boyle, M., Cloutier-Fisher, D., et al. (2009) An interdisciplinary team communication framework and its application to healthcare "e-teams" systems design. BMC Medical Informatics and Decision Making, 9, 43. doi:10.1186/1472-6947-9-43

[5] Meier, D.E. and Beresford, L. (2008) The palliative care team. Journal of Palliative Medicine, 11, 677-681. doi:10.1089/jpm.2008.9907

[6] Mitchell, G., Tieman, J. and Shelby-James, T. (2008) Multidisciplinary care planning and teamwork in primary care. Medical Journal of Australia, 21, S61-S64.

[7] Baldwin, P.K., Wittenberg-Lyles, E., Oliver, D.P. and Demiris, G. (2011) An evaluation of interdisciplinary team training in hospice care. Journal of Hospice \& Palliative Nursing, 13, 172-182. doi:10.1097/NJH.0b013e31820b5c16

[8] National Breast and Ovarian Cancer Centre (2008) Multidisciplinary care principles for advanced disease: A guide for cancer health professionals. National Breast and Ovarian Cancer Centre, Surry Hills.

[9] Benotti, P.N. and Forse, R.A. (1995) The role of gastric surgery in the multidisciplinary management of severe obesity. American Journal of Surgery, 169, 361-367. doi:10.1016/S0002-9610(99)80177-9

[10] Bast Jr., R.C., Kufe, D.W., Pollock, R.E., Weichselbaum, R.R., Holland, J.F. and Frei III, E. (2000) Holland-frei cancer medicine. 5th Edition, BC Decker, Hamilton, 2000.

[11] Valentini, V., Schmoll, H.J. and van de Velde, C.J.H. (2012) Multidisciplinary management of rectal cancerquestions and answers. Springer-Verlag, Berlin and Heidelberg. doi:10.1007/978-3-642-25005-7

[12] Yixinga, J. and Jaffer, A.A. (2010) Multidisciplinary management of gastric cancer. Current Opinion in Gastroenterology, 26, 640-646.

[13] Wetenkamp, V. (2002) Multidisciplinary management: Why me? Clinical Leadership \& Management Review, 16, $175-180$.

[14] Department of Health and Human Services (2013) Multidisciplinary care.

http://www.dhhs.tas.gov.au/cancercare/multidisciplinary_ care

[15] Pittet, D. (2001) Improving adherence to hand hygiene practice: A multidisciplinary approach. Emerging Infectious Diseases, 7, 234-240.

[16] Flaherty, K.R., King Jr., T.E., Raghu, G., Lynch III, J.P., Colby, T.V., Travis, W.D., et al. (2004) Idiopathic interstitial pneumonia: What is the effect of a multidisciplinary approach to diagnosis? American Journal of Respiratory and Critical Care Medicine, 170, 904-910. doi:10.1164/rccm.200402-1470C

[17] Simmonds, S., Coid, J., Joseph, P., Marriott, S. and Tyrer, P. (2001) Community mental health team management in severe mental illness: A systematic review. The British Journal of Psychiatry, 178, 497-502. doi:10.1192/bjp.178.6.497

[18] Webster, S. and Harrison, L. (2004) The multidisciplinary approach to mental health crisis management: An Austra- 
lian example. Journal of Psychiatric and Mental Health Nursing, 11, 21-29.

[19] Vreeland, B. (2007) Bridging the gap between mental and physical health: A multidisciplinary approach. Journal of Clinical Psychiatry, 68, 26-33.

[20] Calles, J.L. (2007) Depression in children and adolescents. Primary Care: Clinics in Office Practice, 34, 243258. doi:10.1016/j.pop.2007.04.008
[21] Baker, L.L. and Ashbourne, L. (2002) Treating child and adolescent depression a handbook for children's mental health practioners, children mental health ontario, chapter 2 multidisciplinary consultation. 11-18.

[22] Desai, N.G., Tiwari, S.C., Nambi, S., Shah, B., Singh, R.A., Kumar, D., et al. (2004) Urban mental health services in India: How complete or incomplete? Indian Journal of Psychiatry, 46, 195-212. 